\title{
Article
}

\section{Multi-Physics Particle Method for the Simulation of Severe Accidents in Nuclear Power Plants}

\author{
Kenta INAGAKI ${ }^{1, *}$ \\ ${ }^{1}$ Central Research Institute of Electric Power Industry, 2-11-1 Iwado Kita, Komae-shi, Tokyo 201-8511, Japan
}

\begin{abstract}
The development of analysis methods for severe accidents in nuclear reactors is a key issue for nuclear safety. It is difficult to estimate the behaviors of several phenomena in reactor accidents, such as the melting and relocation of structural materials, the spreading of corium on the ground, and the molten core concrete interaction (MCCI), because they involve large changes of the geometry. In the present study, a new method was developed to simulate these phenomena by using a moving particle semi-implicit (MPS) method with models for surface tension, rigid bodies, melting and freezing, heat conduction, interfacial heat transfer, and heat radiation. As benchmarks, the melting of a metal cylinder on a hot plate and the freezing of a molten metallic drop in a coolant are simulated. The characteristic behaviors in each experiment agreed well with the simulation results, which indicates that the developed method is applicable for simulation to evaluate the behavior of corium in severe reactor accidents.
\end{abstract}

KEYWORDS: severe accident simulation, MPS method, heat conduction, heat transfer, melting, freezing, rigid, fluid, surface tension

\section{Introduction}

A detailed study of the progress and constituent steps of the reactor accidents that occurred in the Fukushima Daiichi Nuclear Power Plant Units 1-3 of Tokyo Electric Power Co., Inc. and TMI-2 is essential from the viewpoint of ascertaining the status inside the reactor after the accident and determining the measures for prevention of severe accidents in the future ${ }^{1)}$. For an investigation of such severe accidents (SA), an approach for predicting reactor behavior using computer simulation based on the physical phenomena is indispensable. Computational methods offer advantages such as the ability to simulate severe conditions where testing is impossible because of safety concerns, and the ability to evaluate the parameters that are difficult to measure in a test.

The melting and relocation of substances and their solidification are dominant features of various phenomena that occur under severe accident conditions. These include the melting of fuel rods and structural materials (corium), the solidification of corium in the coolant leading to the formation of debris, the spreading behavior of corium on the floor of the reactor, and the molten core concrete interaction (MCCI). Such melting and spreading behavior of corium

\footnotetext{
* Corresponding author, E-mail: inagaki@criepi.denken.or.jp

DOI : 10.15669 /fukushimainsights. Vol.4.417

(C) 2021 Atomic Energy Society of Japan. All rights reserved.

Originally published in Transactions of the Atomic Energy Society of Japan (ISSN 1347-2879), Vol. 14, No. 4, p.249-260

(2015) in Japanese. (Japanese version accepted: July 10, 2015)
} 
strongly influences the progress of phenomena in a reactor accident. On the other hand, physics-based simulation of such phenomena using mesh methods such as the finite element method (FEM) is difficult to apply because any significant change in the positions and shapes of molten substances during the progress of the accident results in heavy computational loads due to the need for remeshing near the surface. For the analysis of a system having such a free liquid surface and significant deformation, the volume of fluid (VOF) method ${ }^{2)}$ or the particle method ${ }^{3)}$ is suitable. However, various phenomena such as heat transfer via radiation, liquid phase flow, solid phase movement, and the interaction of liquid and solid phases have to be taken into consideration. To date, an analysis that considers all of these factors has not been developed. The evaluation of the influence of various conditions and physical property values, such as the temperature and atmosphere inside the reactor, and chemical composition on the behavior of molten substances is possible if an analytical method for these phenomena can be developed. Moreover, such a method makes it possible to predict the important factors affecting individual phenomena. Furthermore, when a simulation experiment is conducted on the individual phenomena that occur as a result of a severe accident, the actual test conditions can be efficiently determined by separately assessing the influence of each condition through a series of analyses with varying parameters. Furthermore, by the modeling of phenomena based on numerical experimental results, a more realistic and effective model can be recommended for the SA general analysis code represented by the Modular Accident Analysis Program (MAAP) ${ }^{4}$ ) or Methods of Estimation of Leakages and Consequences of Releases (MELCOR) ${ }^{5)}$, etc.

Hence, in this study, a simulation code is developed implementing the physical models necessary for analysis of the behavior of molten substances in the reactor. The simulation is aimed at facilitating analysis of the early phase of a reactor accident accompanied by the melting and solidification of substances.

In this approach, the moving particle semi-implicit (MPS) method ${ }^{6}$, a kind of particle method, is adopted as a basis for analysis. Previous research by Koshizuka ${ }^{7)}$ and $\mathrm{Li}^{8)}$, has also applied the MPS method to the analysis of corium in reactor accidents. However, these analyses do not include re-solidification of molten substances by cooling, nor the movement of solid materials. Therefore, they are not suitable for the analysis of a system that is accompanied by the relocation of solid materials. The method by Guo et al ${ }^{9}$.) takes into consideration the movement of the solid materials before melting, but re-solidification of multiple solid bodies and molten droplets are not taken into account. Hence, in the present method, using the MPS method for fluid analysis forms a basis which allows various existing models for solid bodies, surface tension, heat conduction, and melting-solidification to be integrated. Thus, the kinetic behaviors of liquid and solid phases generated through melting-solidification and the interaction between them can be analyzed. Furthermore, this makes it possible to analyze the mobile behavior of corium, with recurring partial melting and solidification. A model for the radiation heat transfer and interfacial heat transfer between solid bodies in the MPS method is newly developed and implemented. In order to verify the feasibility and confirm the precision of the radiation heat transfer model and the interfacial heat transfer model, analysis was conducted on a system with a theoretical solution. Furthermore, simulated calculations for experiments corresponding to the spreading of corium on the floor and corium solidification, i.e., debris generation in the coolant, were analyzed. The feasibility of this code is confirmed by comparison with experimental results, and a discussion is presented on the applicability of the present method to the simulation of individual phenomena in the case of a severe accident. 


\section{MPS Method}

\section{Fluid Analysis Algorithm}

The particle method is a Lagrange method, in which the continuous body is expressed as an aggregate of particles, and the dominant equation is discretized to calculate the forces that work between these particles and the calculated force move the particles. In the MPS method, space derivatives such as the gradient, diffusion, and Laplacian that appear in the dominant equations are discretized using the models in Eqs. (1)-(3) ${ }^{6}$.

$$
\begin{aligned}
& \nabla \phi_{i}=\frac{d}{n^{0}} \sum_{i \neq j}\left[\frac{\phi_{j}-\phi_{i}}{r_{i j}^{2}}\left(\boldsymbol{r}_{j}-\boldsymbol{r}_{i}\right) w\left(r_{i j}\right)\right] \\
& \nabla \cdot \boldsymbol{u}_{i}=\frac{2 d}{n^{0}} \sum_{i \neq j}\left[\frac{\left(\boldsymbol{u}_{j}-\boldsymbol{u}_{i}\right) \cdot\left(\boldsymbol{r}_{j}-\boldsymbol{r}_{i}\right)}{r_{i j}^{2}} w\left(r_{i j}\right)\right] \\
& \nabla^{2} \phi_{i}=\frac{2 d}{\lambda n^{0}} \sum_{i \neq j}\left[\left(\phi_{j}-\phi_{i}\right) w\left(r_{i j}\right)\right]
\end{aligned}
$$

where, $\phi$ and $u$ are physical quantities depicting the scalar and vector, respectively, $i$ and $j$ are the indexes for particles, $d$ is the number of dimensions, $r$ is the position vector and $r_{i j}$ stands for the distance between particles $i$ and $j$. $w$ denotes a weight function that indicates the intensity of interaction between particles. In this study, a newly developed weight function model defined in Eq. (4) is used.

$$
w\left(r_{i j}\right)=\left\{\begin{array}{cc}
\left(\frac{r_{e}}{r_{i j}}-1.0\right) \cdot\left(\frac{r_{0}}{r_{i j}}\right)^{\frac{3}{2}} & r_{i j}<r_{e} \\
0 & r_{i j} \geq r_{e}
\end{array}\right.
$$

where $r_{0}$ is the distance between the two closest particles in the initial scenario where the particles are lined up in a lattice configuration, and $r_{e}$ denotes the influence radius. A larger influence radius will increase the computational load, while a smaller value will produce instability in the computation, such that the optimal value has to be determined while giving consideration to balancing those tendencies. An appropriate influence radius is considered to be $2-4$ times as large as $r_{0}{ }^{6}$ e.g., $r_{e}=2.1 r_{0}$ is frequently used. On the other hand, in a method that deals with the surface tension ${ }^{10)}$, a value 3.2 times as much as $r_{0}$ is recommended. In this study, $r_{e}=3.2 r_{0}$ was adopted because the surface tension is handled. $n^{0}$, appearing in Eqs. (1)-(3) represents the representative particle number density calculated at the inner particle position of the initial configuration, and the particle number density of particle $i$ is defined as the sum of weight functions between all particles $j$ around the particle $i$. Furthermore, $\lambda$ in Eq. (3) denotes a factor for matching of the increase in statistical dispersion and the analytical solution ${ }^{3)}$, and is expressed by the following equation:

$$
\lambda=\frac{\sum_{i \neq j} r_{i j}^{2} w\left(r_{i j}\right)}{\sum_{i \neq j} w\left(r_{i j}\right)}
$$

Tamai et al ${ }^{11)}$. came up with a formulation using the higher order terms that appear in the Taylor expansion of multivariable functions in place of Eqs. (1)-(3). Thereby, they developed a higher order precision differentiation model that can develop the space derivative with an arbitrary precision, taking into account the discretization of the space derivative of the MPS method. However, a concern is that the computation will diverge when the Particle Number Density (PND) condition (described later in this paper) is applied to the pressure source term. 
Therefore, in this study, the conventional discretization model of MPS was adopted.

The dominant equation in the analysis of non-compressive fluid is the Navier-Stokes equation:

$$
\frac{\partial v}{\partial t}=-\frac{1}{\rho} \nabla P+\frac{\mu}{\rho} \nabla^{2} \boldsymbol{v}+\boldsymbol{g}+\boldsymbol{f}
$$

The right-hand side terms denote the forces generated due to the pressure gradient, viscosity, and gravity, and surface tension, respectively. $\rho$ and $\mu$ are the density and viscosity coefficient, respectively. At the first step, only the terms of viscosity, external force, and surface tension are computed by discretizing Eq. (6) into Eq. (7) to obtain the temporal velocity $v^{*}$ and position $r^{*}$.

$$
\begin{aligned}
\boldsymbol{v}_{i}^{*} & =\boldsymbol{v}_{i}+\Delta \mathrm{t}\left[\frac{\mu}{\rho} \frac{2 d}{\lambda n^{0}} \sum_{i \neq j}\left(\boldsymbol{v}_{j}-\boldsymbol{v}_{i}\right) w_{i j}+\boldsymbol{g}+\boldsymbol{f}\right] \\
\boldsymbol{r}_{i}^{*} & =\boldsymbol{r}_{i}+\Delta \mathrm{t} \boldsymbol{v}_{i}^{*}
\end{aligned}
$$

For computation of the viscosity term, the Laplacian model shown in Eq. (3) is used. Additionally, the computation of the surface tension term $f$ that appears in Eq. (7) is explained in the next section.

Next, the pressure at each particle position is computed by using the Poisson equation i.e., Eq. (9), with the particle number density $n^{*}$ and velocity $v^{*}$ computed at the particle position after computation with Eq. (8).

$$
\nabla^{2} P=\alpha \frac{\rho}{\Delta t^{2}} \frac{n^{0}-n^{*}}{n^{0}}+(1-\alpha) \nabla \cdot v^{*}
$$

The first term on the right-hand side of Eq. (9) is the PND (particle number density) condition while the second term stands for the DIV (divergence free) condition. Both of these two are derived from the incompressibility assumption of the system, and only one of the two was used in the conventional MPS method. However, there is a disadvantage in that the variation of numbers in each source term are generally large, and errors tend to accumulate. In the method adopted for this study, Eq. (9) was constructed with reference to the method proposed by Tanaka et al ${ }^{12}$., facilitating stable analysis while avoiding the accumulation of errors under an incompressibility condition by properly adjusting the parameter $\alpha$ between 0 and 1 . In this research, as a result of analyses conducted by varying the value $\alpha$ in each analysis system as explained in sections IV and V, the tendency was confirmed that a larger value of $\alpha$ makes the computation more unstable. On the other hand, if $\alpha$ is set to a value that is too small, the non-compressive condition will collapse (the spatial volume occupied by the particles gradually decreases). Thus, the upper limit for stable computation was defined as $\alpha=0.1$.

After computing the pressure, the movement of particles resulting from the pressure gradient was computed using the following equation, derived from the improved gradient model in Eq. (1):

$$
\nabla P=\frac{d}{n^{0}} \sum_{i \neq j}\left[\frac{P_{j}+P_{i}}{\left(r_{i j}^{*}\right)^{2}}\left(\boldsymbol{r}_{j}^{*}-\boldsymbol{r}_{i}^{*}\right) \cdot w\left(r_{i j}^{*}\right)\right]
$$

The details of deriving Eq. (10) are explained in the report by Oochi et al ${ }^{13)}$. This pressure gradient model is understood to compute the force applied on the particle $i$ from the pressure at the center between particles $i$ and $j$. This makes the computation stable because the resulting force from the pressure gradient is always exhibited as the repulsion force between particles. 


\section{Details of Additional Models}

In this study, the simulation of a sophisticated system including various physical phenomena was made possible by implementing the models of heat conduction, interfacial heat transfer, radiation heat transfer, surface tension, melting and solidification, and rigid body dynamics in the conventional fluid analysis method mentioned in the previous section. An overview of the algorithm of the present method is shown in Figure 1. The items surrounded with a solid frame are the models added to this research, and their details are explained as follows:

\section{(1) Heat conduction}

To conduct a thermal field analysis, the enthalpy per unit volume and temperature $T$ were given for each particle as the variables, similar to the method described by Koshizuka et al ${ }^{6}$. The heat transfer was computed according to the thermal energy preservation law in Eq. (11):

$$
\frac{\partial H}{\partial t}=K \nabla^{2} T+\widetilde{Q}
$$

where, $K$ is the heat conductivity, and $Q$ is the heat source. Furthermore, the enthalpy was set to zero at absolute zero point. Using the Laplacian model of Eq. (3), Eq. (11) is discretized to form Eq. (12).

$$
\frac{\partial H_{i}}{\partial t}=\frac{2 d}{\lambda n_{i}} K \sum_{i \neq j}\left[\left(T_{j}-T_{i}\right) w\left(r_{i j}\right)\right]+\widetilde{Q}
$$

The relation between temperature $T$ and enthalpy $H$ is approximated with Eq. (13):

$$
T_{i}=\left\{\begin{array}{cc}
H_{i} /\left(\rho c_{p}\right) & \left(H_{i}<\rho c_{p} T_{m}\right) \\
T_{m} & \left(\rho c_{p} T_{m} \leq H_{i} \leq \rho c_{p} T_{m}+\rho L\right) \\
\left(H_{i}-\rho L\right) /\left(\rho c_{p}\right) & \left(\rho c_{p} T_{m}+\rho L<H_{i}\right)
\end{array}\right.
$$

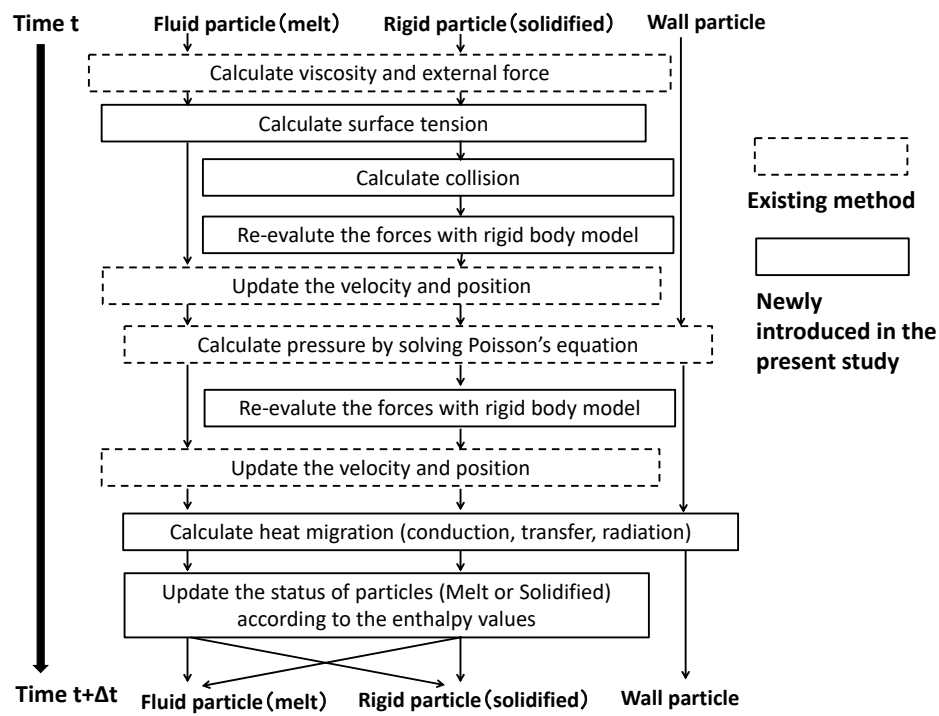

Figure 1 Schematic overview of the algorithm of the proposed method 
where, $T_{m}$ is the melting point, $L$ is the latent heat, and $c_{p}$ is the specific heat. The specific heat was assumed to have no temperature dependence. Furthermore, an identical value was used for the density and specific heat, regardless of the particle conditions (molten or solidified).

\section{(2) Interfacial heat conductance model}

When different solids are in contact, the contact thermal resistance occurs on the interface. The value of contact thermal resistance is influenced to a large extent by the contacting pressure, surface roughness, and heat conductivity of materials. In the present method, the interfacial heat conduction was treated by defining the heat transfer coefficient $h$ between different solids. In the interfacial heat transfer computation, the combination of particles in contact is not detected in particular, but when a particle $j$ belonging to a rigid particle group is detected within the influence radius of particle $i$ belonging to another rigid particle group, the heat transfer $H_{i \rightarrow j}$ between particles $i$ and $j$ is evaluated by the following equation:

$$
H_{i \rightarrow j}=\frac{h}{r_{0}}\left(T_{i}-T_{j}\right) \frac{w_{i j}}{W_{\text {sum }}} d t
$$

where, $W_{\text {sum }}$ is the total sum of the weights function of the entire combination of particles in region A and particles in region B, shown in Figure 2, and is expressed with the following equation:

$$
W_{\text {sum }}=\sum_{i \in A, j \in B} w_{i j}
$$

Using this expression, the heat quantity that moves from A to B through the interface in the center is equal to the theoretical value under the condition shown in Figure 2, where particles are configured in a lattice. To be exact, Eq. (14) is equal to the theoretical value only when the particles are configured in a lattice, but from the viewpoint of reducing the computational load, it was decided to utilize only the value computed with the lattice configuration of particles in the primary stage of computation as $W_{\text {sum }}$.

When either or both of the different phases are melted on the interface, the contact-condition dependent thermal resistance is assumed to be zero, i.e., the heat exchange between all particles that are present in the influence radius is evaluated using Eq. (12) when the particle $i$ is in a molten state. However, the heat conductivity $K$ in this state was set to an average of two particles. On the other hand, when a particle $i$ was solidified and another particle $j$ belonging to

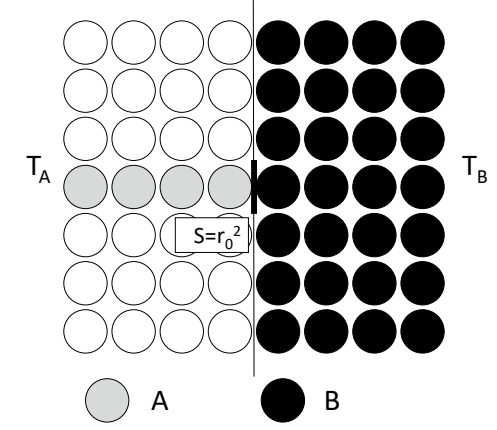

Figure 2 Hypothetical particle geometry on the interface between different phases for the estimation of several parameters 
a different rigid body particle group from particle $i$ was present within its influence radius, Eq. (14) was used for evaluation of the amount of heat exchange between $i$ and $j$.

For the particles located on the surface of the fluid or solid body, heat exchange with the atmosphere is considered. In the present method, no particle was set in the area corresponding to the atmosphere, and thus the method of Eq. (14) was not applicable. Hence, the heat transfer to the atmosphere was considered by varying the heat quantity using Eq. (16) for the surface particles.

$$
\frac{d H_{i}}{d t}=h_{a t m}\left(T_{a t m}-T_{i}\right) / r_{0}
$$

where, $h_{\text {atm }}$ denotes the heat transfer coefficient to the atmosphere, and $T_{\text {atm }}$ denotes the atmospheric temperature.

In order to find a surface particle, a method was adopted that regards the particle for which $n_{i}$ $<\beta n_{0}(\beta \fallingdotseq 0.97)$ holds true as a surface particle, making use of the fact that the value of particle number density decreases near the surface in the conventional particle method. However, the issue was that a local small value of the particle number density on the inside of a continuous body was determined as the surface. Hence, the following method was developed in consideration of the positional information of adjacent particles to avoid erroneous surface detection:

$$
\boldsymbol{a}_{i}=\sum_{i \neq j}\left(\boldsymbol{r}_{j}-\boldsymbol{r}_{i}\right) / r_{i j}
$$

First, the vector $\boldsymbol{a}_{i}$ in Eq. (17) was evaluated. This is the total sum of unit vectors in the direction of the relative positional vector of particle $i$ and adjacent particle $j$. This vector has the property of having a larger absolute value if the location of the adjacent particle is concentrated in a single direction. Using this property, the particle that satisfies one of following three conditions is determined as a surface particle:

(1) $n_{i}<0.875 \cdot n_{0} \cap\left|\boldsymbol{a}_{i}\right|>0.2$

(2) $n_{i}<1.0 \cdot n_{0} \cap\left|\boldsymbol{a}_{i}\right|>0.4$

(3) $n_{i}<0.3 \cdot n_{0}$

The coefficients that appear on the right-hand side are the values adjusted for properly determining surface particles in various shapes.

\section{(3) Radiation model}

For computing the heat transfer by radiation, a surface-to-surface model that assumes the diffusion of gray body thermal radiation was used, where the heat transfer by radiation is computed on the basis of the shape coefficient between each element for all combinations of surface elements in the system. If the surface particles defined by the method in the previous section are used as the surface elements of the surface-to-surface model, the computational load is heavy. In this method, the surface elements were defined differently from the particles used for MPS calculation and evaluation, and radiation heat transfer is calculated between newly defined surface elements, as follows. First, the analysis space in which particles are present was divided by lattice cube geometry. This lattice was termed the surface element extraction lattice. When multiple surface particles were present in a surface element extraction lattice, they were defined as a single surface element (Figure 3). By defining the width of the surface element extraction lattice as a larger size, the load for computation of radiation was decreased. Based on the balance consideration, in this study, the size of the surface element extraction lattice was 


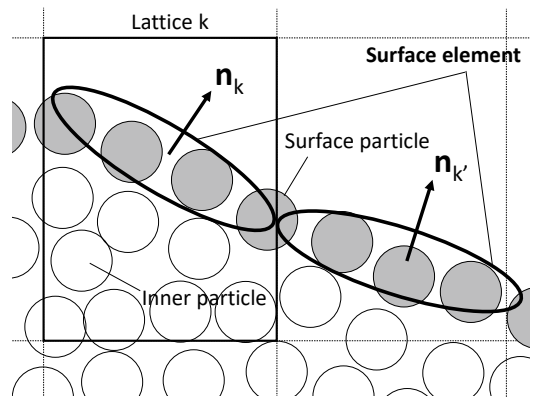

Figure 3 Definition of the surface elements

defined to be of the same dimension as the influence radius, i.e., 3.2 $r_{0}$.

Defining the size of the surface element extraction lattice as $3.2 r_{0}$ was considered to be reasonable because the surface shape was comparatively smooth in the systems analyzed in this study treated in sections IV and V.

The surface area of surface element $S_{k}$ defined in a surface element extraction lattice $k$ is expressed by the following Equation:

$$
S_{k}=r_{0}^{2} \cdot N
$$

where, $N$ is the number of surface particles present in the lattice $k$. Furthermore, the values of position vector $\boldsymbol{x}_{k}$ and normal vector $\boldsymbol{n}_{k}$ of the surface element are defined as the arithmetic average of position and normal vectors of surface particles present in the lattice $k$, respectively. Next, regarding the combination $k$ and $l$ of all surface elements, shape coefficient $F_{k, l}$ was calculated using the following equation:

$$
F_{k, l}=\frac{\cos \theta_{k} \cos \theta_{l}}{\pi r_{k l}^{2}} S_{l}
$$

where, $r_{k l}$ is the distance between surface element $k$ and $l$, and $\theta$ is the angle formed by a line connecting the surface elements and the normal vector. Both of them are computed using the position and the normal vector acquired as described above. For the surface area and the shape coefficient evaluated with Eq. (19) and Eq. (20), several validating evaluations were conducted on some simple primitive shapes. The evaluations confirmed convergence, where smaller particle diameters led each value closer to its theoretical solution.

The quantity of heat released $q_{\text {out }}$ by thermal radiation and incident heat quantity $q_{\text {in }}$ are expressed with the following equations:

$$
\begin{aligned}
& q_{\text {out }, k}=\varepsilon_{k} \sigma T_{k}^{4}+\left(1-\varepsilon_{k}\right) q_{i n, k} \\
& q_{i n, k}=\sum_{l} F_{k, l} q_{\text {out }, l}
\end{aligned}
$$

where, $\varepsilon$ and $\sigma$ are the emissivity and the Stefan-Boltzmann constant, respectively. In each time step, Eq. (21) was computed using $q_{\text {in }}=0$ as the initial value, and the acquired $q_{\text {out }}$ was substituted in Eq. (22). Next, the acquired $q_{i n}$ was substituted in Eq. (21) and such repetition was conducted until convergence was observed. The converged values of $q_{\text {in }}$ and $q_{\text {out }}$ were adopted as the quantity of heat transferred by thermalradiation. The increase or decrease of heat quantity calculated for each surface element was uniformly distributed for surface particles that 
belonged to the surface element.

\section{(4) Surface tension model}

A method using the inter-particle potential (Eq. (23)) developed by Kondo et al ${ }^{10)}$. was adopted for computation of surface tension:

$$
P(r)=C \cdot \frac{1}{3}\left(r-\frac{3}{2} r_{0}+\frac{1}{2} r_{e}\right)\left(r-r_{e}\right)^{2}
$$

The influence of surface tension was considered by applying the inter-particle potential force shown in Eq. (24) between particles $i$ and $j$. The right-hand side of Eq. (24) is a primary spatial differential of Eq. (23).

$$
\rho_{i} r_{0}^{3}\left|\frac{d v_{i}}{d t}\right|=C\left(r_{i j}-r_{0}\right)\left(r_{i j}-r_{e}\right)
$$

The coefficient $C$ in Eqs. (23) and (24) can be derived from Eq. (25) because the energy required for forming two surfaces of area $r_{0}^{2}$ by removing particle group A from particle group B in Figure 2 is equal to $2 \sigma r_{0}^{2}$, thus Eq. (25) is obtained, and then $\mathrm{C}$ was derived.

$$
2 \sigma r_{0}^{2}=\sum_{i \in A, j \in B} P\left(r_{i j}\right)
$$

The potential coefficient $C^{\prime}$ acting between the fluid particle and the wall particle was defined by the following equation:

$$
\frac{C^{\prime}}{C}=\frac{1}{2}(1+\cos \theta)
$$

where, $\theta$ is the contact angle between the fluid and wall. Furthermore, the surface tension coefficient $\sigma_{12}$ that acts between particles in different liquid phases having a surface tension coefficient $\sigma_{1}$ and $\sigma_{2}$, respectively, was defined by the following equation assuming Antonov's rule $^{14)}$ :

$$
\sigma_{12}=\left|\sigma_{1}-\sigma_{2}\right|
$$

\section{(5) Melting and solidification model}

Based on the temperature of particles evaluated using Eq. (13), whether a particle is in a molten or solidified state was determined by the following procedure. First, the particles with temperatures higher than the melting point were regarded to form a molten state, and an ordinary fluid computation process was applied for these. Next, the particles with temperatures lower than their melting points were regarded to form a solidified state, i.e., regarded as rigid body particles. The rigid body model, which is discussed later, was applied to compute the behavior of these particles. If the temperature was equal to the melting point, it was assumed that the particle is partially solidified, and the other part was in a molten state. In the methodology by $\mathrm{Guo}^{9)}$ et al., this state is treated as a fluid body, and the influence of partial solidification is simulated by increasing the viscosity. On the other hand, all the particles at temperatures below their melting point are treated as rigid body particles in this method.

The particles determined as solidified are classified according to the procedure described above into each rigid body particle group. For a cluster forming a solid body, a list is prepared of the particles that belong to it, and the ID of each of these particles is stored.

If solidified particle $i$ at time $t$ already was in a solidified state at time $t-\Delta t$, belonging to 
a rigid particle group, then it is assumed to belong to the same particle group at time $t$ as well. Furthermore, if particle $i$ was in a molten state at time $t-\Delta t$ and is in a solidified state at time $t$, it is regarded as belonging to the same solid particle group of another rigid particle $j$ in the neighborhood, which satisfies Eq. (28), indicating that particles $i$ and $j$ are close enough to each other. If such particle $j$ does not exist, a new solid particle group is defined and particle $i$ is assigned to it. Furthermore, when a particle that was in a solidified state at $t-\Delta t$ and enters a molten state at time $t$, then this particle is deleted from the solid particle group list.

$$
r_{i j} \leq \sqrt{2} r_{0}
$$

Eq. (28) is used for determining whether particles $i$ and $j$ are in contact. It is more natural to define the right-hand side as $r_{0}$, but for some cases the distance between adjacent particles is larger than $r_{0}$ due to the oscillation caused by the pressure gradient between the surrounding particles. For this reason, when the contact determination distance is defined as $r_{0}$, a tendency towards scattered solidified zones was observed. To avoid this, the standard of distance for determining the contact condition of particles was defined as $\sqrt{2} r_{0}$ which is larger than $r_{0}$.

\section{(6) Rigid body model}

The movement of solid particle group $\Pi$ is computed using the following method ${ }^{15)}$ that adopts the quaternion. First, the coordination of the gravity point of a rigid body $\Pi, r_{g, \Pi}$ is expressed as

$$
r_{g, \Pi}=\sum_{i \in \Pi} r_{i} / N_{\Pi}
$$

where, $N_{\Pi}$ is the number of particles that belong to rigid body particle group $\Pi$. Then, the mass of rigid body $M$ and inertia tensor $I$ are acquired with the following equations:

$$
\begin{aligned}
M_{\Pi} & =N_{\Pi} \cdot \rho_{i} r_{0}^{3} \\
\boldsymbol{I}_{\Pi} & =\rho_{i} r_{0}^{3} \sum_{i \in \Pi}\left[\begin{array}{lll}
r_{i g, y}^{2}+r_{i g, z}^{2} & -r_{i g, x} r_{i g, y} & -r_{i g, x} r_{i g, z} \\
-r_{i g, y} r_{i g, z} & r_{i g, x}^{2}+r_{i g, z}^{2} & -r_{i g, y} r_{i g, z} \\
-r_{i g, z} r_{i g, x} & -r_{i g, z} r_{i g, y} & r_{i g, x}^{2}+r_{i g, y}^{2}
\end{array}\right]
\end{aligned}
$$

The $r_{i g}=\left(r_{i g, x}, r_{i g, y}, r_{i g, z}\right)$ in Eq. (31) is the relative position vector of particle $i$ from the reference point of the gravity center $r_{g, I}$. In order to proceed with the rotation in three-dimensional coordinates, a four-dimensional number, named quaternion $Q$, is used. The quaternion at time $t$ is described with the following equation:

$$
\boldsymbol{Q}=\left(s, Q_{x}, Q_{y}, Q_{z}\right)=(1,0,0,0)
$$

The momentum $P_{\Pi}$ and angular momentum $L_{\Pi}$ of rigid body $\Pi$ are given according to the following equation:

$$
\begin{aligned}
\boldsymbol{P}_{\mathrm{II}} & =\sum_{i \in \Pi} \rho_{i} r_{0}^{3} \boldsymbol{v}_{i} \\
\boldsymbol{L}_{\Pi \mathrm{I}} & =\sum_{i \in \Pi} \rho_{i} r_{0}^{3}\left(\boldsymbol{r}_{i g} \times \boldsymbol{v}_{i}\right)
\end{aligned}
$$

Here, the actions by the viscosity term, external force term and surface tension terms are computed using Eq. (7) for all particles that belong to the rigid body $\Pi$, and similarly with other fluid particles. However, the positions acquired from Eq. (8) are not overwritten. Since the 
force acting on particle $i$ can be expressed as

$$
\boldsymbol{F}_{i}=\rho_{i} r_{0}^{3}\left(\boldsymbol{v}_{i}^{*}-\boldsymbol{v}_{i}\right) / d t
$$

the force and torque acting on the rigid body $\Pi$ are obtained in terms of Eqs. (36) and (37) respectively:

$$
\begin{aligned}
& \frac{d \boldsymbol{P}_{\Pi}}{d t}=\sum_{i \in \Pi} \boldsymbol{F}_{i} \\
& \frac{d \boldsymbol{L}_{\Pi}}{d t}=\sum_{i \in \Pi} \boldsymbol{r}_{i g} \times \boldsymbol{F}_{i}
\end{aligned}
$$

Applying the relation of angular momentum and angular velocity $(L=I \omega)$, the angular velocity of the rigid body at time $\mathrm{dt}$ after an action of torque is acquired in the following equation:

$$
\boldsymbol{\omega}=\boldsymbol{I}_{\mathrm{II}}^{-1}\left[\boldsymbol{L}_{\mathrm{II}}+d \boldsymbol{L}_{\mathrm{II}}\right]
$$

where, $\boldsymbol{I}^{-1}$ is an inverse matrix of inertia tensor $\boldsymbol{I}$. The quaternion $\boldsymbol{Q}^{\prime}$ after $d t$ is expressed as,

$$
Q^{\prime}=d Q \times Q
$$

where, $\boldsymbol{d} \boldsymbol{Q}$ denotes the magnitude of rotation that occurs during $d t$, expressed as $\boldsymbol{d} \boldsymbol{Q}=(\cos (\theta / 2)$, $\operatorname{asin}(\theta / 2)$ ), using the rotation axis $a=\omega /|\omega|$ and rotation angle $\theta=|\omega| d t$. The relationship between the rotation matrix $\boldsymbol{R}$ and quaternion is expressed by Eq. (39):

$$
\boldsymbol{R}=\left[\begin{array}{ccc}
1-2 Q_{y}^{2}-2 Q_{z}^{2} & 2 Q_{x} Q_{y}-2 s Q_{z} & 2 Q_{x} Q_{z}+2 s Q_{y} \\
2 Q_{x} Q_{y}+2 s Q_{z} & 1-2 Q_{x}^{2}-2 Q_{z}^{2} & 2 Q_{y} Q_{z}-2 s Q_{x} \\
2 Q_{x} Q_{z}-2 s Q_{y} & 2 Q_{y} Q_{z}+2 s Q_{x} & 1-2 Q_{x}^{2}-2 Q_{y}^{2}
\end{array}\right]
$$

Hence, the position of particle $i$ that belongs to rigid body $\Pi$ after $d t$ is acquired by Eq. (41):

$$
r_{i}^{*}=r_{g, \Pi}+\frac{1}{M_{\Pi}} d \boldsymbol{P}_{\text {II }} d t+\boldsymbol{R} \cdot r_{i g}
$$

Such computation has to be conducted again when computing the pressure gradient term following the computation of pressure.

When there is a collision between two rigid bodies or between a rigid body and the wall, it is treated by permitting a micro-overlap between particles, similar to the discrete element method $(\mathrm{DEM})^{16)}$, and generating a reaction force $f_{c}$ defined by Eq. (42).

$$
\boldsymbol{f}_{c}=-K_{c}\left(\frac{2 m_{i} m_{j}}{m_{i}+m_{j}} \frac{\left(r_{c}-r_{i j}\right)}{d t^{2}}\right) \frac{\left(r_{j}-r_{i}\right)}{r_{i j}}
$$

where, $m$ is the mass of the particle, and $r_{c}$ is the collision distance and equals to $r_{0}$. Furthermore, $K_{c}$ is a factor that determines the magnitude of the reaction force, i.e., the repulsion coefficient, and the value $K_{c}=1.0$ was used for the computation of collision between rigid bodies.

\section{Measures for Stabilizing Computation}

Generally, when the particle number density becomes locally high, the pressure increases, which results in instability in the computation. To prevent this, in the present method, the instability was avoided by specifying a reaction force between fluid particles that have approached each other beyond a certain distance. For computation of reaction force, Eq. (42) was used, 
which is identical to the equation describing the collision between rigid bodies. The collision distance of $r_{c}=0.65 r_{0}$ and the repulsion coefficient of $K_{c}=0.1$ were adopted.

Another instability risk is the zero-pressure of the surface particles, which results in the distance between surface particles becoming too close. Therefore, $r_{c}=0.75 r_{0}$ was adopted only for the case of a collision between surface particles in order to avoid an excessively close distance between surface particles. The collision distance and repulsion coefficient used in these conditions are the ones estimated as most appropriate from the viewpoint of the stability of computation.

\section{Computation for Validation}

\section{Validation Analyses of Heat Conduction and Interfacial Heat Transfer}

As a benchmark analysis of the heat conduction and interfacial heat transfer models introduced in this study, a constant heat conduction analysis on a thick cylinder was conducted using the system shown in Figure $4^{17}$. The temperature distribution in the radial direction was calculated, where the cylindrical area (Area 0$)$ and the cylinder external area $\left(\Omega_{2}\right)$ are regarded as the fixed temperature boundary of $T_{0}=333 \mathrm{~K}$ and $T_{2}=293 \mathrm{~K}$, respectively. The coefficient of heat transfer for boundary $\Omega_{1}$ between Area 0 and Area 1 and the boundary $\Omega_{2}$ between Area 1 and Area 2 were defined as $h_{1}=4,800 \mathrm{~W} / \mathrm{m} / \mathrm{m} / \mathrm{K}$ and $h_{2}=1,500 \mathrm{~W} / \mathrm{m} / \mathrm{m} / \mathrm{K}$, respectively. The heat conductivity in Area 1 was defined as $\lambda_{1}$, and the heat quantity $q_{1}$ that passes through the area in a static state is calculated by the following equation:

$$
q_{1}=\frac{2 \pi\left(T_{0}-T_{2}\right)}{\frac{1}{h_{1} r_{1}}+\frac{1}{\lambda_{1}} \ln \left(\frac{r_{2}}{r_{1}}\right)+\frac{1}{h_{2} r_{2}}}
$$

Hence, the inner wall temperature $T_{\text {in }}$ and outer wall temperature $T_{\text {out }}$ are expressed as follows:

$$
\begin{aligned}
& T_{\text {in }}=T_{0}-\frac{q}{2 \pi r_{1} h_{1}} \\
& T_{\text {out }}=T_{2}+\frac{q}{2 \pi r_{2} h_{2}}
\end{aligned}
$$

Using these equations, the theoretical solution for the inside of the cylinder is:

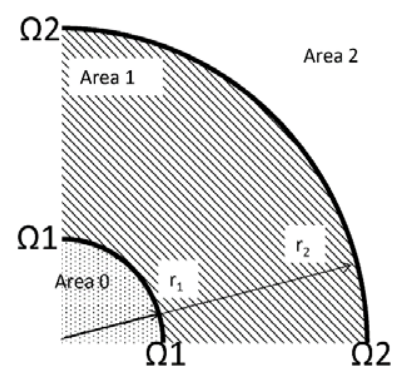

Figure 4 Configuration of the simulation of cylinders for verification of the interfacial heat transfer model 


$$
T=T_{\text {in }}-\frac{\left(T_{\text {in }}-T_{\text {out }}\right) \ln \left(\frac{r}{r_{1}}\right)}{\ln }
$$

A two dimensional analysis was made with 2907 particles where $r_{0}=0.005 \mathrm{~m}$ for configuring the inner and outer cylinders. No particle is placed outside a cylinder. The analysis result of the static state is shown in Figure 5. Furthermore, the comparison of a theoretical solution using Eq. (46) and the computed result is shown in Figure 6. The horizontal axis denotes the distance in the radial direction in Area 1 and the vertical axis denotes the temperature. In a system where the heat transfer coefficient and heat conductivity are dominant, an analysis result that agrees with the theoretical solution was acquired. A good agreement at $r=r_{1}$, where the temperature is strongly influenced by the interfacial heat transfer coefficient $h_{1}$, indicated that the interfacial heat transfer model was reasonable. Similarly, the computation of temperature at $r=r_{2}$ also showed good agreement with the theoretical solution. Consequently, the reasonability of the model of heat transfer to the atmosphere expressed by Eq. (16) was confirmed.

\section{Validation Analysis of Radiation Model}

As a benchmark analysis of the radiation model, computation of thermal migration by radiation between the inner sphere fixed in a space having a radius of $R_{1}=0.003 \mathrm{~m}$ and an outer shell having an inner radius of $R_{2}=0.01 \mathrm{~m}$ was conducted, as shown in Figure 7. The inner surface of the outer shell was defined as the fixed temperature boundary of $T_{2}=873.15 \mathrm{~K}$, and the initial temperature of inner sphere $T_{1,0}$ was defined as $1673.5 \mathrm{~K}$. Finally, the change in temperature of the inner sphere due to heat migration by radiation was computed.

The heat conductivity of the inner sphere was assumed to be sufficiently high, and the

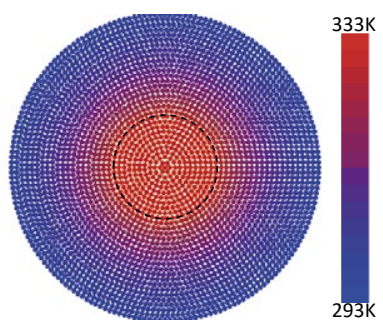

Figure 5 Temperature distribution in the simulation of cylinders in terminal static state

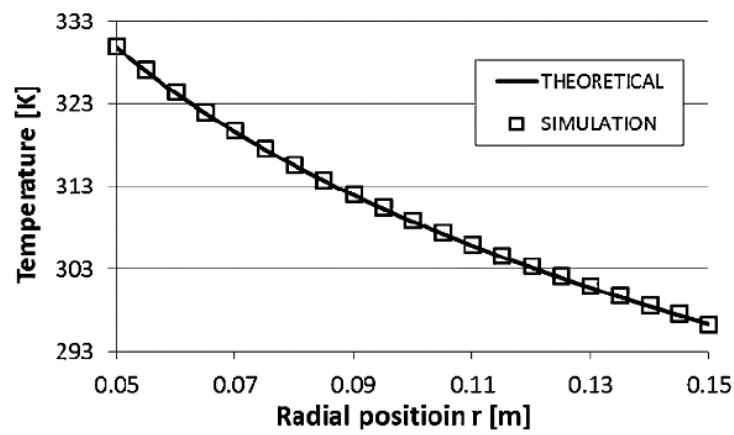

Figure 6 Comparison of theoretical and simulaiton results for temperature simulation in cylinders 
temperature distribution in the inner sphere was assumed as always uniform. The relationship between time $t$ and the inner sphere temperature $T_{1, t}$ is expressed by the following equation ${ }^{18)}$ :

$$
t=\frac{\rho V C_{p}\left[1 / \varepsilon_{1}+\left(A_{2} / A_{1}\right)\left(1 / \varepsilon_{2}-1\right)\right]}{A_{2} \sigma} \times\left[\frac{1}{4 T_{2}^{3}} \ln \left|\frac{\left(T_{1, t}+T_{2}\right) /\left(T_{1, t}-T_{2}\right)}{\left(T_{1,0}+T_{2}\right) /\left(T_{1,0}-T_{2}\right)}\right|+\frac{1}{2 T_{2}^{3}}\left(\tan ^{-1} \frac{T_{1, t}}{T_{2}}-\tan ^{-1} \frac{T_{1,0}}{T_{2}}\right)\right]
$$

where, $A_{1}$ and $A_{2}$ denote the surface area of the inner sphere and inner surface area of outer shell, respectively; $\rho, V$, and $C_{p}$ are the density, volume, and specific heat, respectively, of the inner sphere. The system analyzed with the present method is shown in Figure 8. The green particles denote the surface of the inner sphere and the red particles denote the inner surface of the outer shell. The particle diameter was $0.00035 \mathrm{~m}$, the total number of particles was 49,525, and $\mathrm{dt}$ was defined as $0.01 \mathrm{~s}$. The time progression of the inner sphere temperature acquired

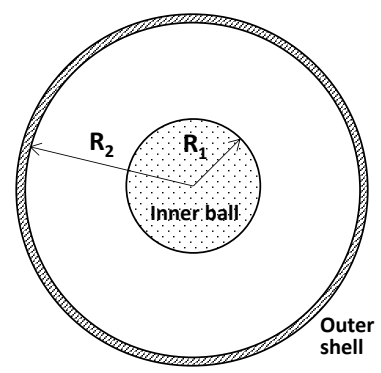

Figure 7 Simulation configuration of a sphere within an outer shell for verification of the heat radiation model

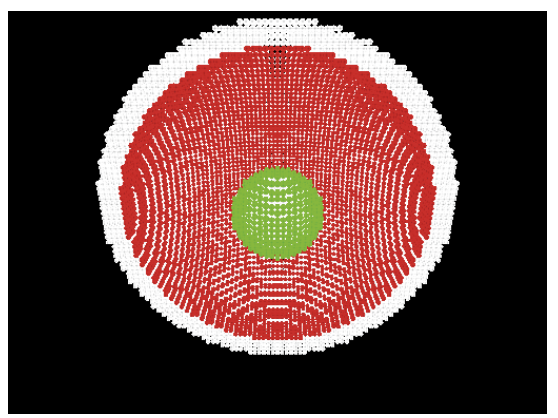

Figure 8 Configuration of the particles for the simulation of a sphere within an outer shell.

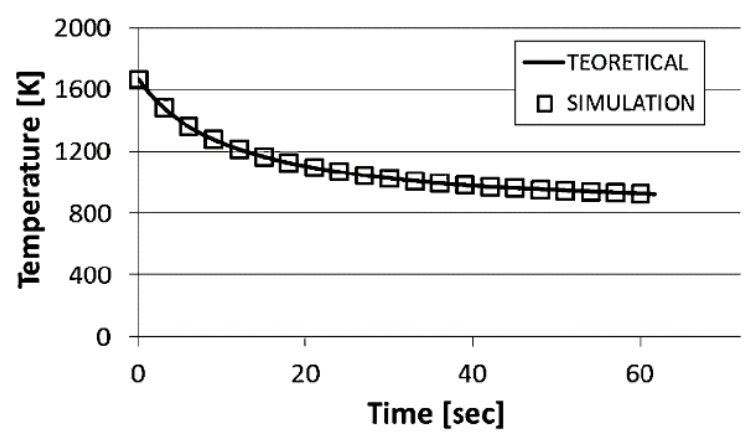

Figure 9 Comparison of theoretical and simulation results of the temperature behavior of the sphere 
by the analysis is shown in Figure 9 along with the theoretical solution using Eq. (47). The theoretical solution was consistent with the analysis results, confirming the reasonability of the radiation model developed in this study.

\section{Analysis of Cylinder Melting Test}

\section{Model and Conditions}

For the analysis of spreading of molten materials on the floor in a nuclear reactor accident, it is essential to conduct a study of a system where surface tension can be taken into consideration to a reasonable extent, since it has a significant influence on fluid behavior. Hence, as a benchmark for the present method, the analysis of a cylinder melting test described by Furuya et al ${ }^{19)}$. was conducted. In the test, a tin cylinder was placed on a $0.0025 \mathrm{~m}$ thick alumina plate in an air atmosphere held at a constant temperature, and the melting migration process was observed from three directions. The initial temperature of the alumina plate and air atmosphere was $655 \mathrm{~K}$. The tin cylinder was $0.005 \mathrm{~m}$ high, and its diameter was $0.02 \mathrm{~m}$. The physical properties of the materials used for the analysis are shown in Table 1. The heat transfer from the surface of the materials to the air atmosphere was defined as $25 \mathrm{~W} / \mathrm{m}^{2} / \mathrm{K}$, which is a typical value if air with natural convection ${ }^{20)}$ is assumed. According to Yuan et al ${ }^{21)}$., the contact angle between molten tin and alumina is approximately $160^{\circ}$, but this is a value in an environment where the oxygen partial pressure is $8.56 \times 10^{-6} \mathrm{MPa}$, and it has been reported that the contacting angle has the tendency to decrease with an increase in the oxygen partial pressure. Because this analysis was conducted in an air atmosphere, the contacting angle was assumed to have decreased and was determined as $150^{\circ}$. Furthermore, when determining the interfacial heat transfer coefficient between the tin cylinder and the alumina plate before melting, the analyses were conducted with a modified interfacial heat transfer coefficient and adjusted to acquire the same melting start time between test and analysis, as the point at which melting of the tin cylinder was set to begin was originally $1 \mathrm{~s}$ after the start of the cylinder melting test. The analyses were repeated while increasing the interfacial heat transfer coefficient by 1,000 and adopted a heat transfer of $32,000 \mathrm{~W} / \mathrm{m}^{2} / \mathrm{K}$, as the closest melting start time to the test, $1.1 \mathrm{~s}$, was acquired by the analysis using this value. In the analysis performed in this study, only the above-mentioned interfacial heat transfer coefficient was used as an adjusting parameter, while other physical properties were adopted from the literature.

In a cylinder melting test analysis using this method, there was a situation where only the particles on one perimeter of the cylinder bottom were melted immediately after melting of the cylinder started. When the pressure became zero because molten particles were determined as surface particles, a phenomenon where they were pulled into the inside of a rigid body because an attraction force due to surface tension potential was applied from the surrounding rigid body particles was observed. To avoid this, the collision distance was defined as $r_{c}=0.85 r_{0}$ only for the collision computation of fluid particles and rigid body particles. The coefficient of restitution was defined as $K_{c}=0.1$ similarly to the collision between fluid particles. All other numerical analysis parameters were adopted from those described in section II.

The parameters relevant to the particle method analysis are shown in Table 2.

The tin cylinder and alumina plate models were formed into a three-dimensional particle model. The size of the tin cylinder was designed to correspond to the actual shape and size of the physical cylinder. On the other hand, the alumina plate used for the test was a square shape of $0.1 \mathrm{~m} \times 0.1 \mathrm{~m}$, while from the viewpoint of decreasing the computational load, a disc shape 
Table 1 Physical parameters for the simulation of the melting of a metallic cylinder on a hot plate

\begin{tabular}{|c|c|c|}
\hline Physical parameters & Tin & Alumina \\
\hline Initial temp. $[\mathrm{K}]$ & 300 & 655 \\
\hline Melting temp. $[\mathrm{K}]$ & 505 & - \\
\hline Density $\left[\mathrm{kg} / \mathrm{m}^{3}\right]$ & 7,000 & 4,000 \\
\hline Heat conductivity (fluid) $[\mathrm{W} / \mathrm{m} / \mathrm{K}]$ & 30.0 & - \\
\hline Heat conductivity (solid) $[\mathrm{W} / \mathrm{m} / \mathrm{K}]$ & 66.6 & 30.0 \\
\hline Heat capacity $[\mathrm{J} / \mathrm{kg} / \mathrm{K}]$ & 228.4 & 780.0 \\
\hline Latent heat $[\mathrm{J} / \mathrm{kg}]$ & 59,600 & - \\
\hline Surface tension $[\mathrm{N} / \mathrm{m}]$ & 0.5 & - \\
\hline $\begin{array}{l}\text { Contact angle of the tin on the } \\
\text { alumina [degrees] }\end{array}$ & \multicolumn{2}{|c|}{150} \\
\hline Kinematic viscosity $\left[\mathrm{m}^{2} / \mathrm{sec}\right]$ & $1.3 \times 10^{-7}$ & - \\
\hline $\begin{array}{l}\text { Heat transfer coefficient (air) } \\
{\left[\mathrm{W} / \mathrm{m}^{2} / \mathrm{K}\right]}\end{array}$ & 25 & 25 \\
\hline $\begin{array}{l}\text { Heat transfer coefficient between solid } \\
\text { tin and alumina }\left[\mathrm{W} / \mathrm{m}^{2} / \mathrm{K}\right]\end{array}$ & \multicolumn{2}{|c|}{32,000} \\
\hline
\end{tabular}

Table 2 Particle parameters for the simulation of the melting of a metallic cylinder on a hot plate

\begin{tabular}{ccc}
\hline & Tin cylinder & Alumina plate \\
\hline Number of particles & 6,776 & 15,764 \\
Thickness $[\mathrm{m}]$ & 0.005 & 0.0025 \\
Diameter $[\mathrm{m}]$ & 0.02 & 0.04 \\
Particle size $\mathrm{r}_{0}[\mathrm{~m}]$ & \multicolumn{2}{c}{0.000625} \\
dt $[\mathrm{sec}]$ & \multicolumn{2}{c}{$2.0 \times 10^{-4}$} \\
\hline
\end{tabular}

of diameter $0.045 \mathrm{~m}$ was adopted for analysis. The total number of particles was 22,540 .

\section{Analysis Result}

A comparison of the results acquired by the analysis and testing on cylinder melting are shown in Figure 10 and the results are depicted in Table 3. In the experiment, the melting initiated at $1 \mathrm{~s}$ after start, and a change in the height was confirmed at $10 \mathrm{~s}$. The melting of the cylinder was complete after $18 \mathrm{~s}^{19}$. In the simulation, on the other hand, melting started at 1.1 s. Following this, the change in height and melting of the entire cylinder was observed at 9.5 $\mathrm{s}$ and $18.1 \mathrm{~s}$, respectively. Hence, this proved that the test results were simulated with good precision. Viewing the cylinder section in the simulation result, the melting of the cylinder was confirmed to have started from the periphery of the surface, contacting with the alumina plate and spreading to the upper side and in the cylinder center direction. Furthermore, the visible change in height was confirmed to have occurred at $9.5 \mathrm{~s}$, caused by the pushing out of the molten zone of the lower part to the outside due to compression by the remaining solid zone in the upper part after all particles in the bottom surface of cylinder had melted. The molten zone of the cylinder maintained the oval shape due to surface tension.

The interfacial heat transfer from the alumina plate to the tin cylinder was dominant immediately after test start. After this, the heat transfer to the tin cylinder was restricted by the heat conduction from the periphery of the alumina plate to the center zone, as the temperatures of the tin cylinder and the alumina plate in the zone immediately below the cylinder were almost equal after $9.5 \mathrm{~s}$ when a change in height of the cylinder was observed. In this analysis, the air flow around the cylinder was not computed, and a simplified model was used instead 


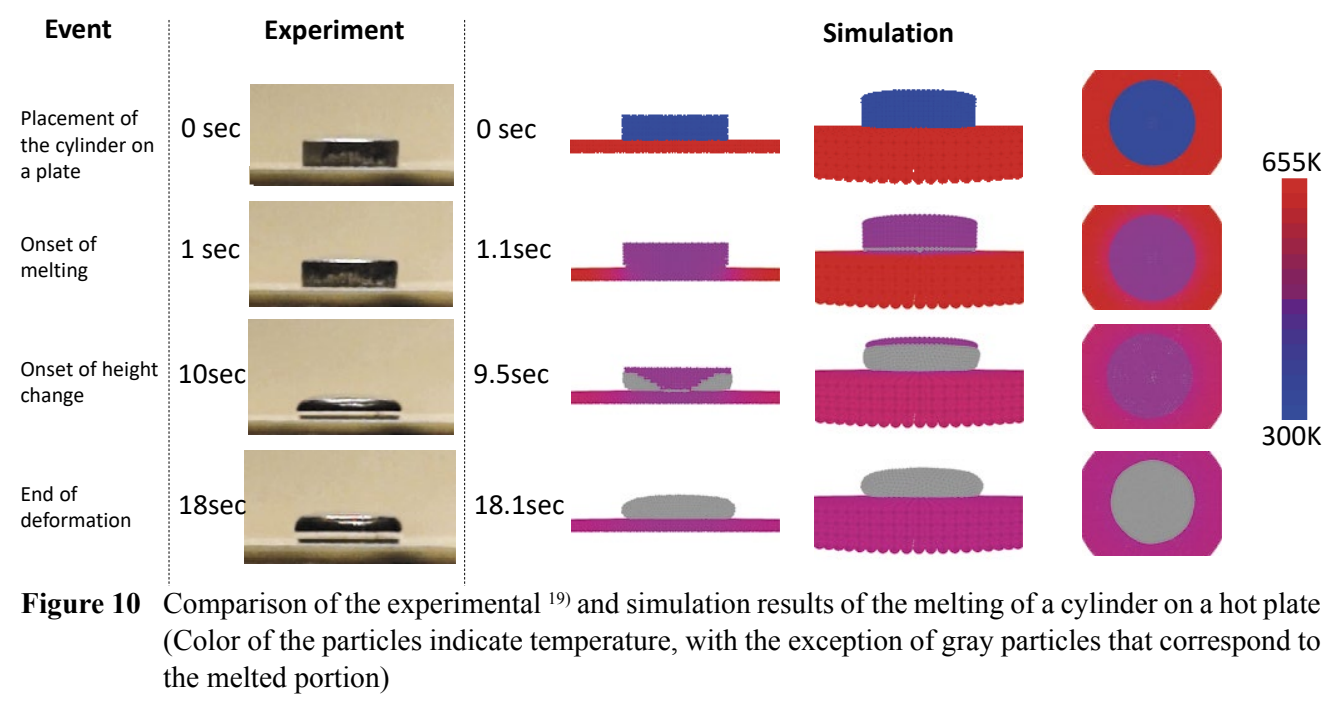

Table 3 Chronology of key phenomena in the experiment and the simulation

\begin{tabular}{ccc}
\hline & Experiment & Simulation \\
\hline Onset of melting & 1 & 1.1 \\
Onset of height change & 10 & 9.5 \\
End of deformation & 18 & 18.1 \\
\hline
\end{tabular}

that calculated the heat exchange between the cylinder surface and air with a constant heat transfer coefficient. The amount of heat exchange between the cylinder surface and air was sufficiently smaller than that from the alumina plate, and the influence was assumed to be miniscule. Regarding the comparison of shape, the experimental result was reproduced well by the simulation. The visual contact angle of less than $150^{\circ}$ was explained to be due the influence of weight of the molten tin, etc.

It was confirmed that the melting could be analyzed with a reasonable degree of precision when the influence of surface tension or the phase transition is dominant. Given that this system is the behavior of fluid aggregation by surface tension on the floor, this is a phenomenon resembling the spreading of corium on the floor as part of the elementary process of a reactor accident. As the corium spreads across the floor, reduced friction with floor due to the formation of a gaseous phase at the interface between the corium and concrete floor may occur. This method may be applicable to the analysis of such corium spreading behavior by introducing a model that accurately simulates the corium-concrete reaction.

On the other hand, a part of the melting phenomena that may occur in a reactor accident is the eutectic reaction such as Zircalloy $/ \mathrm{UO}_{2}, \mathrm{Ni} / \mathrm{Zr}$, etc. To handle this, it is necessary to consider the formation of a liquid phase that spreads with the speed controlled by the reaction between different types of substances. However, because the formation of a liquid phase area and spreading occurs on a smaller scale than the typical length of flux, it is not realistic to directly model the eutectic reaction for analysis from the viewpoint of computation load. As a solution to this issue, a method that includes the modeling of the liquid phase quantity as a function defined by time, temperature and the particle configuration may be adopted. By introducing a proper eutectic reaction model, this code will be also be applicable to the melting 
phenomena of fuel and cladded tubes.

\section{Solidification Analysis of Debris in Coolant Material}

\section{Analysis Models and Analysis Conditions}

As a benchmark for a system that includes different types of liquid phases, analysis was conducted on a testing system that simulated the solidification process of a molten substance dropped in the coolant ${ }^{22}$. For the test, a molten droplet of aluminum was dropped in a sodium pool in a stainless steel cylindrical container (I.D. $0.108 \mathrm{~m}$ and $0.3 \mathrm{~m}$ deep), and the shape of collected solidified aluminum was observed. The weight of the aluminum liquid droplet was about $5 \mathrm{~g}$, and it was dropped from a height of $0.18 \mathrm{~m}$ from the surface of the sodium pool. The physical parameters of the analysis are shown in Table $4^{22}$. Three different temperatures $960 \mathrm{~K}, 1,100 \mathrm{~K}$, and $1,200 \mathrm{~K}$ were adopted as the initial temperature of the aluminum droplet. This analysis focused on a range of conditions where boiling of sodium does not occur, and the initial temperature of the aluminum droplet was set within a range where the sodium temperature does not exceed the boiling temperature.

The parameters relevant in the particle method analysis are shown in Table 5. This analysis was conducted in two dimensions and the particle diameter was $0.001 \mathrm{~m}$. The total number of particles, including the wall particles, was 35,756. An aluminum droplet of diameter $0.02 \mathrm{~m}$ was set immediately above the sodium liquid surface and an initial downward speed of $2.0 \mathrm{~m} / \mathrm{s}$ was specified in the analysis. The parameters that are not listed in Table 5 are all adopted from the values explained in section II, and no adjustment of the parameters specific to debris solidification analysis was made.

Table 4 Physical parameters for the simulation of a molten metallic droplet being dropped into a coolant pool

\begin{tabular}{ccc}
\hline Physical parameters & $\mathrm{Al}$ & $\mathrm{Na}$ \\
\hline Initial temp. [K] & $960,1,100$, or 1,200 & 540 \\
Melting temp. [K] & 933 & - \\
Density $\left[\mathrm{kg} / \mathrm{m}^{3}\right]$ & 2,357 & 885 \\
Heat conductivity (fluid) $[\mathrm{W} / \mathrm{m} / \mathrm{K}]$ & 97.4 & 78.0 \\
Heat conductivity (solid) $[\mathrm{W} / \mathrm{m} / \mathrm{K}]$ & 238 & - \\
Heat capacity [J/kg/K] & 1,080 & 1,310 \\
Latent heat $[\mathrm{J} / \mathrm{kg}]$ & 395,000 & 115,000 \\
Surface tension $[\mathrm{N} / \mathrm{m}]$ & 0.878 & 0.179 \\
Kinematic viscosity $\left[\mathrm{m}^{2} / \mathrm{sec}\right]$ & $4.3 \times 10^{-6}$ & $4.3 \times 10^{-6}$ \\
Heat transfer coefficient $(\mathrm{air})\left[\mathrm{W} / \mathrm{m}^{2} / \mathrm{K}\right]$ & 25 & 25 \\
\hline
\end{tabular}

Table 5 Particle parameters for the simulation of a molten metallic droplet being dropped into a coolant pool

\begin{tabular}{ccc}
\hline & Al droplet & Na pool \\
\hline Number of particles & 305 & 32,591 \\
Particle size $\mathrm{r}_{0}[\mathrm{~m}]$ & \multicolumn{2}{c}{0.001} \\
dt $[\mathrm{sec}]$ & \multicolumn{2}{c}{$2.0 \times 10^{-5}$} \\
\hline
\end{tabular}




\section{Analysis Results and Discussion}

The three analysis results with different initial aluminum droplet temperatures are shown in Figure 11. In the simulation results on the right side of chart, the red particles depict aluminum in a molten state, while the black particles depict aluminum in a solidified state, and the gray particles depict the sodium. The results obtained with all initial temperatures exhibited significant deformation of the aluminum droplet that plunged into the sodium pool, due to compression by the inertia and the repulsion from the sodium pool. For the case where the initial temperature of the droplet was $960 \mathrm{~K}$, the entire bottom part of the aluminum droplet solidified due to aluminum-sodium heat transfer.

Resultantly, a cup-like crust of the aluminum drop was formed. After that, the upper part was cooled by contact with sodium and finally the whole drop solidified into a cup-like shape. On the other hand, in the case where the initial temperature of the droplet was $1,100 \mathrm{~K}$, a crust covering the entire bottom area as observed at the initial temperature of $960 \mathrm{~K}$ was not formed. The aluminum droplet separated into several masses and fragmented due to the tensile viscous forces, etc., accompanying the sodium flow. Also in the case where the initial temperature of the droplet was $1,200 \mathrm{~K}$, the tensile forces caused similar behaviors. In this case, separation into even smaller fragments occurred. For comparison, the solidified aluminum fragments obtained in the experiment are shown on the left side of Figure 11. In the experiment, when the temperature of the aluminum droplet was comparatively low $(1,131 \mathrm{~K})$, a solidified substance having a cup-like shape was formed, and when the temperature was high $(1,272 \mathrm{~K})$, branch-like and fine particle solids were formed. These results closely correspond to the appearance of the shapes acquired in the simulation. Furthermore, the sizes of solidified substances were in rough agreement between experiment and the simulation. Thus, the analysis results by the present method reproduced the phenomenon well. On the other hand, the cup-like solidified substances were generated at $960 \mathrm{~K}$, while the branch-like and fine particles were generated at initial droplet temperatures of 1,100 and $1,200 \mathrm{~K}$ in the simulation. These temperatures were comparatively

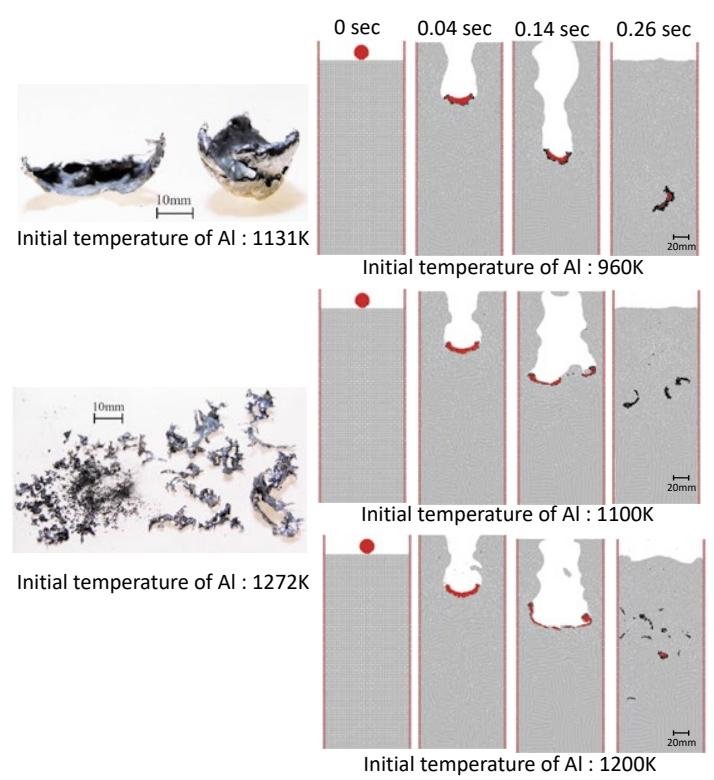

Figure 11 Appearance of the frozen Al droplet obtained in the experiment ${ }^{22)}$ (left) and the result of the simulation (right). Black: Al (Solidified), Red: Al (melt), Gray: Na (melted) 

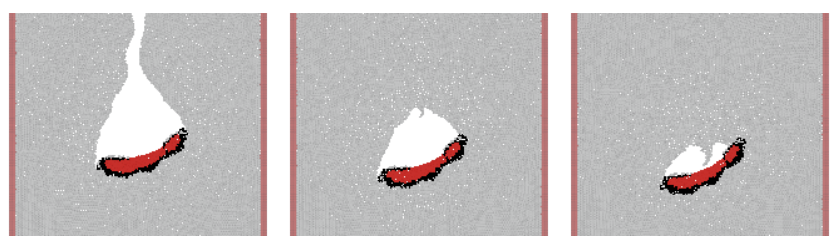

Figure 12 Appearance of the sodium jet leaping down from the sodium pool surface

lower than those in the experiment. This can be explained, as the initial temperature acquired in the experiment was measured in the upper part of the melting crucible, while the temperature decreased when collision occurred with the sodium pool, possibly due to heat exchange with the air during the fall, etc.

In the test ${ }^{22}$, a tip with a large curvature factor is formed when the sodium liquid surface rejoins after separation caused by the collision with the aluminum droplet, and a sodium jet caused by the surface tension acting at this location was observed on the liquid surface. This sodium jet is assumed to have similarly occurred in a downwards vertical direction. In this analysis, the reproduction of this downward sodium jet was confirmed as well (Figure 12).

Because the qualitative analysis of the generation of the aluminum solidified substance was successful, the analysis of solidification behavior of the metal droplet that plunged into the coolant under a range of conditions confirmed that it was not accompanied by the boiling of the coolant. Therefore, this code is expected to be similarly applicable to the analysis of the behavior of portions of corium that are generated in the reactor. Furthermore, this analysis was conducted in two dimensions in order to reduce the computational load; however, the stability and the reasonability of computation is expected to remain intact when expanded to three dimensions. Significant results could be generated by conducting three-dimensional analyses on systems that are difficult to reduce to two dimensions, such as non-symmetrical shapes.

In this analysis, the range of initial temperatures of the aluminum droplet focused on the range where the initial temperature is lower than the boiling temperature of sodium in this analysis. However, when the contact temperature of the droplet and sodium pool exceeds the boiling temperature of sodium, the evaporation of sodium and the generation of a steam film may dominate the phenomenon. Considering application to the early phase of a nuclear reactor accident, boiling of the coolant (water) in a light water reactor may influence the progress of the phenomenon. Furthermore, no particle is positioned in the air zone in this analysis, and only the heat transfer between the surface of a substance and the atmosphere is considered as an influence of the atmosphere. In an actual situation, the influence of air sucking on the sodium flux is considered to exist. As a future subject for analysis of such conditions, the evaporation of liquid and the motion of gas have to be taken into consideration.

\section{Conclusions}

The models of heat transfer, interfacial heat transfer, heat radiation, melting/solidification, rigid body, and surface tension were implemented in the MPS method code. The newly developed heat radiation model and interfacial heat transfer model in this study have been analyzed in a system with a theoretical solution, and good agreement between analysis results and theoretical values was confirmed.

As a benchmark analysis of the present method, the cylinder melting test and the debris 
solidification test in the coolant were simulated, and good reproduction of the phenomena was realized in each case. The analysis of phenomena such as the spreading of corium on the floor is possible by the present method, provided properly modeled phenomena that are assumed to occur in corium-concrete are implemented for simulation of the early phase of a reactor accident. Furthermore, with respect to debris solidification in the coolant, a more meticulous analysis is possible by implementing a model that takes into account the influence of the generation of gas phase, etc.

We would like to take this opportunity to express our sincerest gratitude to Mr. Takanari Ogata, Mr. Satoshi Nishimura, and Mr. Masahiro Furuya of the Central Research Institute of Electric Power Industry for valuable advice and comments on this study.

\section{References}

1) Division of Nuclear Fuel Molten Fuel Sub-Working Group, "Status and issues on nuclear fuel research in the field of LWR severe accident," J. Nucl. Sci. Technol., 56[9], (2014). [in Japanese]

2) C. Hirt, B. D. Nichols, "Volume of fluid method for the dynamics of free boundaries," J. Computat. Phys., 39, 201-225 (1981).

3) S. Koshizuka, Y. Oka, "Moving-particle semi-implicit method for fragmentation of incompressible fluid," J. Nucl. Sci. Eng., 123, 421-434 (1996).

4) Fauske and Associates, LLC., MAAP5-Modular Accident Analysis Program User's Mannual, 2, Part $1-3(2008)$.

5) R. O. Gauntt, R. K. Cole, M. Erickson et al., MELCOR Computer Code Manuals, Primer and Users' Guide, Version 1.8.5, NUREG/CR-6119, 1, Rev. 2 (2000).

6) S. Koshizuka, Ryuushi-hou Simulation, Maruzen (2005).

7) S. Koshizuka, J. Liu, N. Shirakawa et al., "R\&D of the next generation safety analysis methods for fast reactors with new computational science and technology (1)-(6)," Proc. 16th Int. Conf. on Nucl. Eng. (ICONE16), Orland, May 11-15 (2008).

8) X. Li, Y. Oka, "Numerical simulation of the SURC-2 and SURC-4 MCCI experiments by MPS method," Ann. Nucl. Energy, 73, 46-52 (2014).

9) L. Guo, Y. Kawano, S. Zhang, T. Suzuki, K. Morita, K.Fukuda, "Numerical simulation of theological behavior in melting metal using finite volume particle method," J. Nucl. Sci. Technol., 47[11], 1011-1022 (2010).

10) M. Kondo, S. Koshizuka, M. Takimoto, "Surface tension model using inter-particle potential force in moving particle semi-implicit method," Trans. JSCES, Paper No. 20070021, (2007). [in Japanese]

11) T. Tamai, K. Shibata, S. Koshizuka, "Development of the higher-order MPS method using the taylor expansion," Trans. JSCES, Paper No. 20130003, (2013). [in Japanese]

12) M. Tanaka, T. Masunaga, "Stabilization and smoothing of pressure on MPS method by quasi-compressibility,” Trans. JSCES, Paper No. 20080025, (2008). [in Japanese]

13) M. Oochi, S. Koshizuka, M. Sakai, "Explicit MPS algorithm for free surface flow analysis," Trans. JSCES, Paper No. 20100013, (2010). [in Japanese]

14) A. Robledo, C. Varea, "Wetting transition for the contact line and antonov's rule for the line tension," Phys. Rev. A, 45[4], 2423-2428 (1992).

15) M. Tanaka, M. Sakai, S. Koshizuka, "Particle-based rigid body simulation and coupling with fluid simulation,” Trans. JSCES, Paper No. 20070007, (2007). [in Japanese]

16) P. A. Cundall, O. D. L. Strack, "A discrete numerical model for granular assemblies," Geotechnique, 29, 47-65 (1979).

17) K. Washidu, Yuugenyouso-hou Handbook, Baifu-kan, Tokyo, ISBN 978-4563031800 (1983). [in Japanese]

18) H. T. Kim, B. W. Rhee, J. H. Park, "Benchmark calculations of a radiation heat transfer for a CANDU fuel channel analysis using the CFD code," J. Nucl. Sci. Eng., 43[11], 1422-1430 (2006).

19) M. Furuya, "Development of numerical method for fuel rod melting ((1) Experimental observation)," Proc. of Konso-ryu Symposium, Nagano, Aug. 9-11 (2013). [in Japanese]

20) Y. Katsuto, Den-netsu Gairon, Youken-do, (1964). [in Japanese]

21) Z. Yuan, K. Mukai, K. Takagi, M. Ohtaka, "Dependence of surface tension of molten tin on temperature and oxygen partial pressure," J. Jpn. Inst. Metals, 65[1], 21-28 (2001). [in Japanese] 
22) S. Nishimura, I. Kinoshita, N. Ueda et al., "Study on transformation and fragmentation behavior of molten metal in sodium pool," CRIEPI Rep., L03 (2005). [in Japanese] 